\title{
Hydrophilic molecularly imprinted nanospheres for the extraction of rhodamine B followed by HPLC analysis: A green approach and hazardous waste elimination
}

\author{
Maryam Arabi ${ }^{\mathrm{a}}$, Abbas Ostovan ${ }^{\mathrm{a}, *}$, Ahmad Reza Bagheri $^{\mathrm{b}}$, Xiaotong Guo ${ }^{\mathrm{a}, \mathrm{e}}, \mathrm{Jinh} \mathrm{Bu}_{\mathrm{Li}}^{\mathrm{a}}$, \\ Jiping $\mathrm{Ma}^{\mathrm{c}}$, Lingxin Chen ${ }^{\mathrm{a}, \mathrm{d}, * *}$ \\ ${ }^{\text {a }}$ Key Laboratory of Coastal Environmental Processes and Ecological Remediation, Research Center for Coastal Environmental Engineering and Technology, Yantai Institute \\ of Coastal Zone Research, Chinese Academy of Sciences, Yantai, 264003, China \\ ${ }^{\mathrm{b}}$ Chemistry Department, Yasouj University, Yasouj, 75918-74831, Iran \\ ${ }^{\mathrm{c}}$ School of Environmental \& Municipal Engineering, State-Local Joint Engineering Research Center of Urban Sewage Treatment and Resource Recovery, Qingdao University \\ of Technology, Qingdao, 266033, China \\ ${ }^{\mathrm{d}}$ Department of Chemistry and Chemical Engineering, Qufu Normal University, Qufu, 273165, China \\ ${ }^{\mathrm{e}}$ University of Chinese Academy of Sciences, Beijing, 100049, China
}

\section{A R T I C L E I N F O}

\section{Keywords:}

Molecularly imprinted polymers

Green synthesis

Eco-friendly waste

Matrix solid phase dispersion

Experimental design

Rhodamine B

\begin{abstract}
A B S T R A C T
In this work, hydrophilic molecularly imprinted nanospheres (MINs) were synthesized via surface imprinting technology and subsequently utilized as dispersant sorbent in matrix solid phase dispersion (MSPD) for the extraction of rhodamine $\mathrm{B}(\mathrm{RhB})$ as the illogical food additive dye from different foodstuffs, followed by HPLC analysis. By considering hydrophilicity of target analyte, the sol-gel route was chosen for the preparation of MINs in aqueous media at mild conditions. Most importantly, both synthesis and extraction steps were designed and performed in the line of green chemistry and hazardous waste was eliminated to minimize detrimental impact on the operator health and the environment. The reputable experimental design methodology was adopted to assist the condition optimization of MSPD, which comprehends the significance of the factors and their interactions with the least experimental runs. Under optimized MINs-MSPD-HPLC conditions, the detection limit of RhB was down to $0.14 \mu \mathrm{g} \mathrm{kg}^{-1}$ and excellent linearity over the wide range of $0.5-10000 \mu \mathrm{kg}^{-1}$ was attained. Furthermore, endogenous RhB was found in the tested food samples with relative standard deviations (RSDs) of $\leq 4.6 \%$, and satisfactory recoveries were from 83.6 to $96.9 \%$. The simple green MINs-MSPD method holds great potential for determination of trace $\mathrm{RhB}$ in complicated solid/semi-solid samples, showing rapidity, accuracy and reliability.
\end{abstract}

\section{Introduction}

Rhodamine $\mathrm{B}(\mathrm{RhB})$ is a hydrophilic red azoic synthetic dye belonging to a methyl-xanthene group and widely used in food industries [1]. However, often eating foodstuffs containing RhB easily leads to accumulating this colorant in the human and animal digestive system and causes uncomfortableness, various diseases or even endanger life [2]. Concerns about side effects of RhB have begun, since the carcinogenicity and toxicity of this colorant was experimentally proved. Hence, some countries such as the USA and China have banned the use of RhB in foodstuffs and classified it as an illegal colorant (China Food and
Drug Administration, http://www.sfda.gov.cn/WS01/CL0001/). Despite the legal prohibition, usage of RhB has been continued in secret due to the cheapness and bright color, which could have a serious threat to human health. As a result, quantitative analysis of RhB at trace levels in foods is essential. In this regard, influential sample pretreatment should be adopted before instrumental analysis to minimize matrix interferences and enrich RhB concentration to attain highly sensitive detection.

In analytical measurement, a sample pretreatment step is very critical since most of the systematic and random errors occur during this step; and meanwhile, it usually occurs that a large volume of chemicals

\footnotetext{
* Corresponding author.

${ }^{* *}$ Corresponding author. Key Laboratory of Coastal Environmental Processes and Ecological Remediation, Research Center for Coastal Environmental Engineering and Technology, Yantai Institute of Coastal Zone Research, Chinese Academy of Sciences, Yantai, 264003, China.

E-mail addresses: saman.ostovan@yahoo.com (A. Ostovan), 1xchen@yic.ac.cn (L. Chen).
} 
and organic solvents are consumed and hazardous wastes are generated. Therefore, performing simple, lower toxic, cheap and efficient sample pretreatment is highly demanding. Owing to the advantages of matrix solid phase dispersion (MSPD) such as high throughput, effective clean-up, compatibility with solid and semi-solid samples, lower solvent consumption, and shorter extraction time [3], we have been encouraged to employ MSPD as an influential sample preparation technique for RhB analysis. Different from most of the sample preparation methods, in MSPD, the extraction process doesn't occur in liquid media, and analyte's mass-transfer is accomplished by blending and grinding the sample with dispersant sorbent in a mortar [4]. In the meantime, two fundamental drawbacks are along with this technique. One is that co-adsorption of matrix impurities on the surface of dispersant sorbent is difficult to be removed by washing solvent properly, which causes unreliable analytical results. The other is that after an extraction procedure is finished, the mixture of sample and dispersant sorbent extremely blended should be discarded and thereby a high volume of garbage is generated. Commonly, the selected samples for analysis are environmental/food samples and don't pollute the ecosystem when returned to the environment, but dispersant sorbents could seriously pollute the surroundings since they aren't bio-degradable and consist of dangerous chemicals. For addressing the mentioned drawbacks in the line of green chemistry, we purposed to synthesize selective and ecofriendly dispersant sorbent for MSPD.

Molecularly imprinted polymers (MIPs) have gained popularity as selective sorbents, being versatile tailor-made receptors with predefined recognition sites to distinguish target molecules from analogous compounds [5]. Unfavorably, traditional MIPs suffer from fundamental restrictions such as incomplete template removal, slow mass transfer, poor site accessibility, and low binding capacity. Surface imprinting is a creative strategy in which the specific cavities are located onto or near the surface of the imprinted polymers and could significantly dominate MIPs' limitations. Usually, thin MIPs layer is anchored on the surface of diverse materials [6-10] which are selected according to the functionality, cost, specific surface area, and toxicity. Carbon sphere (CS) has attracted wide interest as an excellent support material due to its exceptional properties such as cheap and wide available natural precursor, multifunctionality, high surface area, low density, physical and chemical stability, eco-friendliness, and excellent biocompatibility [11]. To the best of our knowledge, in all reported CS based MIPs, toxic organic precursors, large volume organic solvent and temperature controlling are required for preparation of MIPs layer [12-14]. These kinds of MIPs could pollute the ecosystem while leaking to the environment and have a detrimental impact. On the other hand, during the extraction process, adsorption of non-polar matrix impurities like lipids and proteins on the surface of hydrophobic acrylic based MIPs can hinder the mass-transfer of small template analytes and decrease extraction efficiency [15].

Sol-gel reactions can be performed in aqueous or alcoholic media at mild conditions without the protection of inert gas as well as the toxicity of its precursors is remarkably lower than organic reagents. Moreover, sol-gel based MIPs have obviously higher selectivity towards the target species, and allow faster diffusion than that of acrylic based MIPs [16]. Most importantly, the silica based materials are widely regarded as green, bio-compatible, and eco-friendly ones.

Therefore, herein, we prepared non-toxic and eco-friendly hydrophilic molecularly imprinted nanospheres (MINs) by the sol-gel route and surface imprinting technology for MSPD of RhB followed by HPLC determination. The MINs were well characterized by Fourier transforminfrared spectroscopy (FT-IR), scanning electron microscopy (SEM) and transmission electron microscopy (TEM), and then were employed as a selective dispersant sorbent of MSPD. Several variables affecting MSPD efficiency were systematically investigated, with the aid of central composite design and response surface. The MINs-MSPD-HPLC method was well validated and successfully applied to the determination of RhB in chili powder, tomato ketchup, and jelly powder samples.

\section{Experimental}

\subsection{Materials and instruments}

All chemicals and reagents including glucose, 3-aminopropyl trimethoxysilane (APTMS), RhB, rhodamine 6G, alizarin, chrysoidine pure powders, tetraethyl orthosilicate (TEOS), methanol, ethanol, ethyl acetate, ammonium hydroxide, acetone, hexane, and acetic acid were obtained from Merck (Darmstadt, Germany). Deionized water used throughout the work was ultrapure water obtained from a Milli-Q water purification system (Millipore, Bedford, MA, USA). Chili powder, tomato ketchup, and jelly powder samples were purchased from the local supermarket and kept in the refrigerator at $+4{ }^{\circ} \mathrm{C}$ until use.

Scanning electron microscope (SEM), transmission electron microscope (TEM), Fourier transform-infrared spectroscopy (FT-IR) and high performance liquid chromatography (HPLC) instruments were the same as that of our previous literature [17]. The HPLC mobile phase consisted of a deionized water-methanol (80:20, v/v) mixture which was filtered through a $0.45 \mu \mathrm{m}$ filter, followed by degassing under vacuum and passed with the flow rate of $1.0 \mathrm{~mL} \mathrm{~min}^{-1}$. The separation process was carried out at the ambient temperature and detector wavelength was set at $546 \mathrm{~nm}$.

\subsection{Preparation of MINs}

First, CS was obtained by the carbonization of glucose in aqueous media using an economic hydrothermal route according to our previous work [18]. Then, MINs were synthesized by a green approach as follows: $400 \mathrm{mg}$ of CS was dispersed in $20 \mathrm{~mL}$ of deionized water with ultrasonic vibration for $15 \mathrm{~min}$. $1.75 \mathrm{mmol}$ of RhB, $2 \mathrm{~mL}$ of APTES (8.55 mmol), $4 \mathrm{~mL}$ of TEOS (10.08 mmol), and $400 \mu \mathrm{L}$ of acetic acid $\left(1.0 \mathrm{~mol} \mathrm{~L}^{-1}\right)$ were sequentially added to the mixture and kept away for completing the polymerization at room temperature for $24 \mathrm{~h}$ under a continuous stirring. Afterward, the particles were separated from the solution by centrifugation and rinsed several times with $5 \%$ acetic acid solution in order to complete template removal. Finally, the as-prepared products were rinsed with deionized water thoroughly and dried in the vacuum. The corresponding non-imprinted nanospheres (NINs) were prepared with the same protocol except in the absence of RhB.

\subsection{Adsorption experiments}

Adsorption experiments were carried out to assess the adsorption capacities of MINs and NINs. Typically, $10 \mathrm{mg}$ of MINs or NINs was suspended in $15 \mathrm{~mL}$ of RhB solution at different initial concentrations (5-50 mg L ${ }^{-1}$ ). After shaking for $12 \mathrm{~h}$ at $25{ }^{\circ} \mathrm{C}$, the sorbents were separated by centrifugation, and residual concentrations of RhB were determined by HPLC. The amount of RhB adsorbed by the MINs/NINs was calculated according to the following equation:

$Q=\left(C_{0}-C_{t}\right) V / m$

where $Q\left(\mathrm{mg} \mathrm{g}^{-1}\right)$ is the sorption amount of $\mathrm{RhB}, C_{0}\left(\mathrm{mg} \mathrm{mL}^{-1}\right)$ is the initial concentration of $\mathrm{RhB}, C_{\mathrm{t}}\left(\mathrm{mg} \mathrm{mL}^{-1}\right)$ is the supernatant concentration of RhB after adsorption, $V(\mathrm{~mL})$ is the total volume of sample solution, and $m$ is the mass of the sorbent used.

\subsection{MINs-MSPD of RhB from foodstuffs}

To investigate the applicability of the developed method, three widely using foodstuffs with different matrices, namely chili powder, tomato ketchup and jelly powder were selected as the tested samples. The extraction and clean-up process occurred concurrently as follows: $0.05 \mathrm{~g}$ of each sample and $0.1 \mathrm{~g}$ of MINs were disgorged in a tiny mortar and thoroughly blended and homogenized with a pestle. The fine mixture was transferred into the centrifuge tube and $3 \mathrm{~mL} n$-hexane was added as washing solvent followed by shaking for $2 \mathrm{~min}$. Then, the 


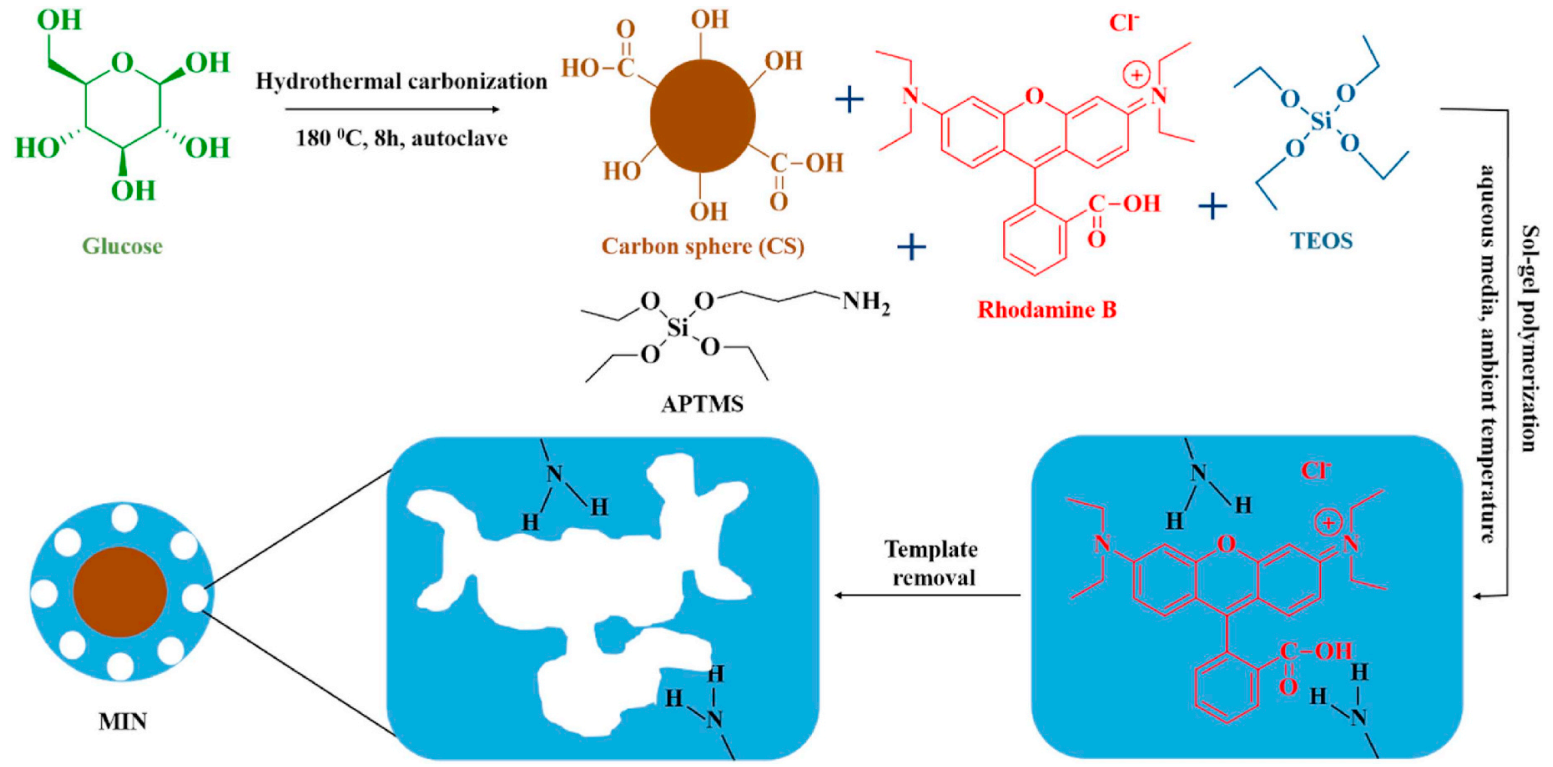

Fig. 1. Schematic illustration for the basic preparation procedure of MINs.

materials were separated by centrifugation and the supernatant was decanted. In the next, the retained $\mathrm{RhB}$ was desorbed by re-dispersing MINs into $3 \mathrm{~mL}$ methanol/acetic acid (90:10, v/v) by shaking. Finally, the eluent was collected in a vial and evaporated in a water bath at $45{ }^{\circ} \mathrm{C}$ under nitrogen stream, and then the residue was dissolved in a $50 \mu \mathrm{L}$ mobile phase and $20 \mu \mathrm{L}$ of it was injected to the HPLC. The MSPD protocol for three types of samples was the same as above, except tomato ketchup in which $0.2 \mathrm{~g}$ of $\mathrm{Na}_{2} \mathrm{SO}_{4}$ was added. Tomato ketchup is a viscous liquid sample and has a high content of water. Herein, $\mathrm{Na}_{2} \mathrm{SO}_{4}$ was added as a drying agent and dried the water of the sample and facilitated the homogenization. Two other samples were solid and no drying agent was needed.

\section{Results and discussion}

\subsection{Preparation of MINs}

Fig. 1 schematically illustrates the synthesis process of MINs, which was divided into two main steps wholly in accordance with the principles of green chemistry. First, green CS was created from natural, cheap, wide available and multifunctional precursor of glucose by hydrothermal route. Glucose is a hydrophilic monosaccharide that can easily dissolve in water. While the aqueous solution of glucose is situated in an autoclave under pressure and temperature, it can be converted to versatile solid globular materials with unparalleled properties. Interestingly, the hydrophilicity and functional groups of glucose are preserved by CS, which makes CS easily uptake hydrophilic analyte and also have -OH wealthy surface that provides sufficient sites for further chemical modification. Most importantly, because CS originates from a natural source, it is non-toxic, eco-friendly, economic, and biocompatible. Meanwhile, all the generated wastes were clean and not hazardous for the environment since the carbonization occurred in aqueous media. In the second step, the eco-friendly hydrophilic inorganic MIPs layer was grafted on the surface of CS via the sol-gel route. As is well known, fabrication of non-covalent imprinted materials in aqueous systems is still challengeable [19], owing to the by-effect of water that seriously interferes with the interactions between functional monomer and template. In other words, in the water-rich systems, the number of water molecules is much higher than that of the template and functional monomer, and they can easily act as both acceptors and donors of hydrogen bonds. Since the water molecules compete to break hydrogen bonds, sustainable pre-complex of template and functional monomer cannot be produced.

On the other hand, most of the prepared MIPs consist of hydrophobic raw reagents namely alkenyl monomer and cross-linker, leading to a remarkable discount of the diffusion of a hydrophilic analyte. Besides, large amounts of toxic organic porogen and hazardous hyperactive organic functional monomers are used for the preparation of MIPs, which drastically injure operator health and pollute the environment. However, sol-gel reactions (hydrolysis and condensation) can easily perform in water media with supreme imprinting efficiency sans of interference of water's by-effect [20]. Since RhB is a water soluble compound, efficient extraction can be only obtained by hydrophilic sorbent according to the "like dissolves like" law. In this regard, APTMS and TEOS were selected as functional monomer and crosslinker, respectively. It is well known that both acid and base can accelerate the sol-gel process. On the other hand, the imprinting efficiency of MIPs is extremely dependent on the durability of $\mathrm{RhB}$ and APTMS pre-complex. At acidic $\mathrm{pH}$ below 6.5, the $\mathrm{NH}_{2}$ group of APTMS is in the protonated form $\left(\mathrm{NH}_{3}{ }^{+}\right)$, while at $\mathrm{pH}$ values higher than 3.2 , a carboxylic acid group of $\mathrm{RhB}$ induces a negative charge and transfers to carboxylate species which can interact with APTMS via the strong electrostatic bond and form well-matched pre-complex. Therefore, acetic acid (acidic media) was used as a catalysis in the sol-gel reaction. After polymerization in aqueous media at ambient temperature, the template molecules were removed from MINs network and a well-designed specific sorbent for trapping RhB was obtained. Alike CS, preparation of the MIPs layer performed in aqueous media without dangerous rubbish, especially silica based materials are environmentally friendly and bio-compatible [21,22]. By the criterion for selection of these precursors and polymerization route, not only green chemistry in imprinting technology were satisfied, but also the cost of materials and the feasibility of MINs synthesis conditions in an industrial scale were considered.

\subsection{Characterization of MINs}

FT-IR was used for the identification of functional groups of CS and MINs (Fig. 2a). The characteristic bands at 1704 and $1618 \mathrm{~cm}^{-1}$ could be assigned to the $\mathrm{C}=\mathrm{O}$ and $\mathrm{C}=\mathrm{C}$ vibration, respectively. The broad absorption band was observed at $3404 \mathrm{~cm}^{-1}$ and related to the high content of O-H groups on the CS surface. For MINs, the peaks at 1090 and $800 \mathrm{~cm}^{-1}$ represented asymmetric and symmetric stretching vibrations of Si-O, respectively. Notably, the characteristic peak of $\mathrm{NH}_{2}$ 

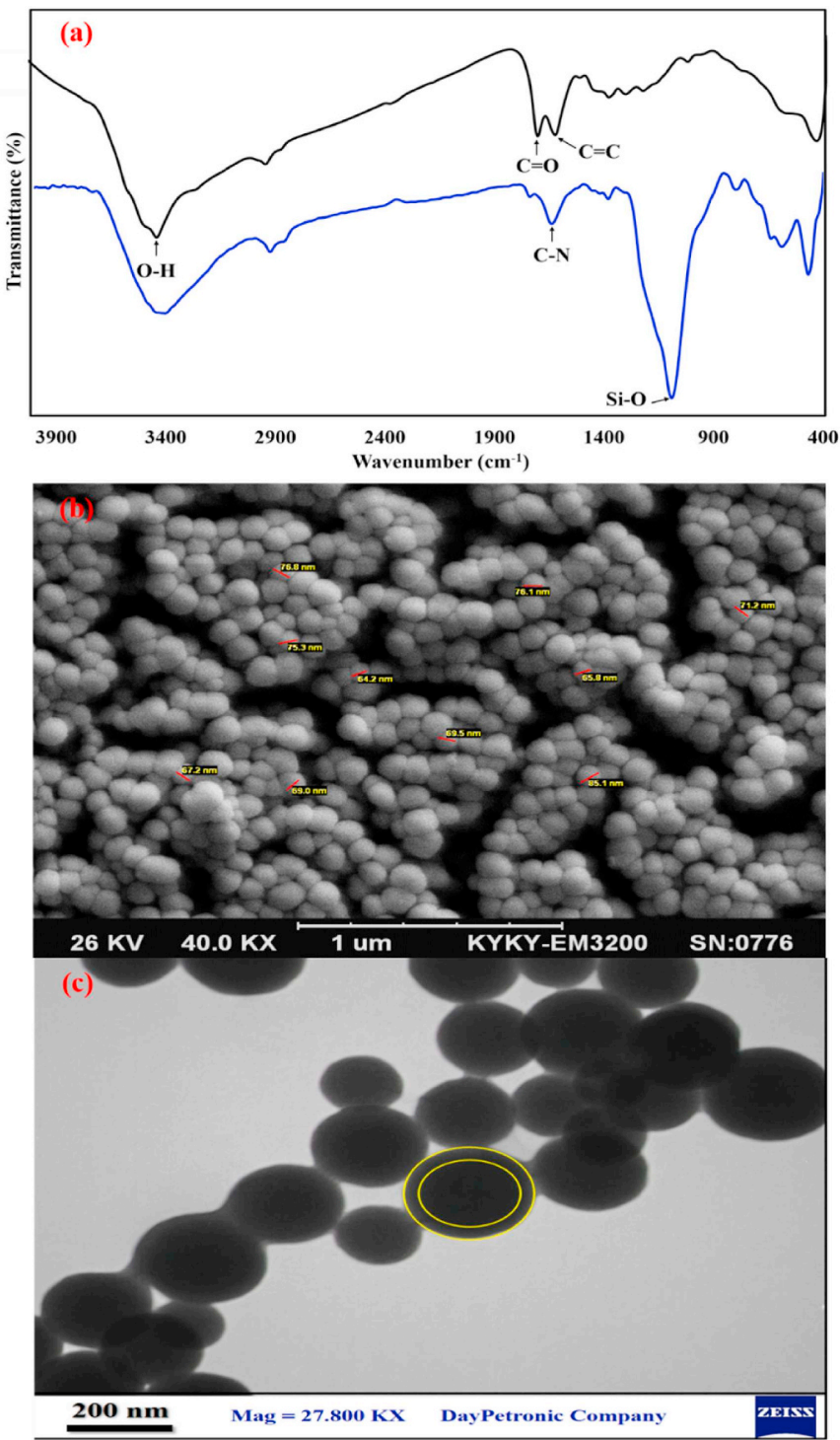

Fig. 2. (a) FT-IR spectra of CS and MINs (black and blue lines related to CS and MINs, respectively), (b) SEM image of MINs, and (c) TEM image of MINs. (For interpretation of the references to color in this figure legend, the reader is referred to the Web version of this article.)

groups at about $1393 \mathrm{~cm}^{-1}$ and the aliphatic stretching of the $\mathrm{C}-\mathrm{H}$ bond emerged at $2954 \mathrm{~cm}^{-1}$, suggesting that amine groups could feasibly interact with RhB. The broad absorption band at around $3500 \mathrm{~cm}^{-1}$ in MINs spectra, corresponded to the hydroxyl group of MINs and adsorbed water molecule on/into the silica network [23]. The intensity peaks of $\mathrm{C}=\mathrm{O}$ and $\mathrm{C}=\mathrm{C}$ in MINs significantly lower than $\mathrm{CS}$ revealed that the support was thoroughly decorated by the MIPs layer.

The shape and morphology of the MINs were well investigated by SEM and TEM. SEM image showed the regular spherical shape of material with relatively identical size distribution (Fig. 2b). Moreover, MINs had a nanoscale diameter (below $100 \mathrm{~nm}$ ) structure, in favor of the full removal of template, which would produce high adsorption capacity and accessible specific cavities facilitating mass-transfer of the $\mathrm{RhB}$. The same results were also observed from the TEM image, which obviously demonstrated the uniform globular configuration and coreshell structure (Fig. 2c). As seen, the thickness of the imprinted layer (light region) was approximately $30 \mathrm{~nm}$, uniformly coating the surface of the CS (dark region). Such thin layer of polymer is sufficient for the influential binding of the target analyte, and meanwhile, the too thin layer is fragile and easily broken during blending the MINs and sample.
These observations proved the successful synthesis of MINs beads.

\subsection{Binding study of the MINs for RhB}

The adsorption isotherm is a substantial aspect of adsorption studies that illustrate the tendency, recognition ability and adsorption capacity of the sorbent. Therefore, binding isotherms of RhB onto MINs and NINs were investigated in the concentration range of $5-50 \mathrm{mg} \mathrm{L}^{-1}$. As can be seen from Fig. S1a, adsorption capacities of MINs and NINs increased by enhancing the initial concentration of $\mathrm{RhB}$ and then were saturated at $40 \mathrm{mg} \mathrm{L}^{-1}$. The maximum adsorption capacity for MINs $\left(37 \mathrm{mg} \mathrm{g}^{-1}\right)$ was higher than NINs $\left(8.8 \mathrm{mg} \mathrm{g}^{-1}\right)$, which verified the important role of template in the creation of specific cavities in the MINs structure. Generally, in the core-shell MIPs, the core capsulated by the MIPs layer and relatively unavailable for template recognition [24]. Even, if a slight amount of analyte penetrated into the core materials, the sorption mechanism is non-specific, since the core materials don't have specific binding sites. The adsorption capacity defined as the bonded amount of analyte per unit mass of sorbent. Therefore, by using low-density and porous CS as core [25], most of the mass of the MINs is related to MIPs shell and the adsorption capacity of the sorbent can be increased significantly. To evaluate the binding features of MINs and NINs, Langmuir and Freundlich isotherm models were used, as presented in Table S1. As can be seen from the table, Freundlich isotherm was the better model since it had $R^{2}$ higher than 0.99 (Fig. S1b). Freundlich isotherm assumes that the adsorption of analyte molecules occurs onto the heterogeneous surface of sorbent [26]. By comparing the $m$ values of MINs and NINs, NINs's $m$ is higher than that of MINs, revealed homogeneity binding sites of NINs [27]. This phenomenon can be explained by the existing two types of binding sites in the MINs network. The analyte molecules can be adsorbed with specificity by imprinted cavities, and/ or non-selectively adsorbed on the surface of MINs. In contrast, only weak and nonspecific adsorption occurred for RhB by NINs.

To assess the binding property and affinity of MINs toward RhB, Scatchard analysis was also used according to the equation at below [28]:

$\frac{Q}{C_{e}}=\left(\frac{Q_{m}-Q}{K_{d}}\right)$

where $Q$ is the adsorbed RhB onto MINs, $C_{\mathrm{e}}$ is the RhB concentration at equilibrium, $Q_{\mathrm{m}}$ is the apparent maximum binding amount, and $K_{\mathrm{d}}$ is the dissociation constant of the adsorption sites; $1 / K_{\mathrm{d}}$ namely $K_{\mathrm{a}}$ is the association (affinity) constant of the adsorption sites. As presented in Fig. S1c, MINs had two different lines while NINs had one line, which revealed the presence of two different kinds of adsorption sites in MINs while only one kind of adsorption site in NINs network, respectively [29]. The Scatchard parameters of MINs including $K_{\mathrm{a}}, K_{\mathrm{d}}$, and $Q_{\mathrm{m}}$ were obtained to be $3.0712 \mathrm{mg} \mathrm{L}^{-1}, 0.325 \mathrm{mg} \mathrm{L}^{-1}$ and $24.97 \mathrm{mg} \mathrm{g}^{-1}$ for the specific (high-affinity) binding sites, respectively, and $0.3684 \mathrm{mg} \mathrm{L}^{-1}$, $2.71 \mathrm{mg} \mathrm{L}^{-1}$ and $42.49 \mathrm{mg} \mathrm{g}^{-1}$ for the non-specific (low-affinity) binding sites, respectively, while $K_{\mathrm{a}}, K_{\mathrm{d}}$, and $Q_{\mathrm{m}}$ of NINs were achieved to be $0.0246 \mathrm{mg} \mathrm{L}^{-1}, 40.65 \mathrm{mg} \mathrm{L}^{-1}$ and $19.58 \mathrm{mg} \mathrm{g}^{-1}$ according to the slopes and intercepts of the obtained plots, as listed in Table S2.

\subsection{Selectivity examination of MINs}

An ideal MIPs can specifically uptake the target analyte and distinguish it from analog compounds. Various factors affecting MIPs' selectivity such as the affinity of functional monomer toward template, the type of porogen, and the synthesis condition should be considered in designing MIPs. To appraise the selectivity, the binding capacity, imprinting factor (IF), and selectivity factor (SF) of MINs for RhB and three similar compounds namely rhodamine 6G, alizarin and chrysoidine (with their chemical structures given in Fig. S2) were compared. As seen in Fig. 3, the adsorption capacities of MINs for rhodamine 6G, 


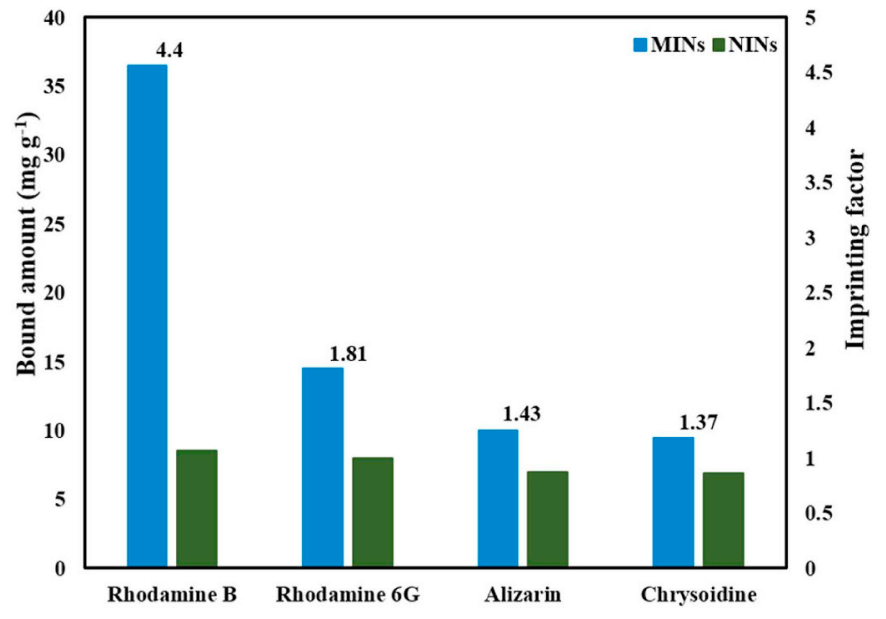

Fig. 3. Selectivity of the MINs and NINs for RhB over three analogs including rhodamine $6 \mathrm{G}$, alizarin, and chrysoidine.

alizarin and chrysoidine were $14.5,10.0$, and $9.5 \mathrm{mg} \mathrm{g}^{-1}$, respectively. Meanwhile, the adsorption capacity of NINs for all the species was approximately identical. Hence, it can be concluded that the created cavities in the MINs are remarkably matched to the RhB. By comparing the size and functional groups of analog compounds, it can obviously see Rhodamine $6 \mathrm{G}$ has the most resemblance to RhB. Owing to this similarity, Rhodamine $6 \mathrm{G}$ molecules can more facilely enter into the imprinted cavities, compared to other similar compounds and attain higher adsorption capacity. For chrysoidine and alizarin, large distinction in the shape, volume, and functional groups' position led to increased steric hindrance unfitting in specific cavities. Nevertheless, the adsorption capacity for alizarin, was a bit more than chrysoidine which may be due to the approximate similarity of alizarin with the upper part of the RhB. Hereupon, the combination of stereochemistry and functionality made a substantial contribution to the selectivity of MINs. The IF values of MINs for RhB, rhodamine 6G, alizarin, and chrysoidine, were $4.4,1.81,1.43$ and 1.37 , respectively, indicating a noteworthy achievement of MINs for hydrophilic molecular recognition of the RhB. Altogether, the results demonstrated that the imprinting process was smoothly performed in aqueous media and the resulting MINs had the excellent selectivity for RhB.

\subsection{Condition optimization of MINs-MSPD for RhB}

Uttermost extraction yield and accurate analytical results can only be attained at genuinely optimum conditions. The main effective variables are divided into qualitative and quantitative ones. In order to attain an optimization approach, qualitative parameters are preliminarily investigated by univariate optimization, and quantitative factors are studied by multivariate optimization. In this work, related optimization experiments of MINs-MSPD for RhB were carried out by using a spiked blank sample at the concentration of $500 \mu \mathrm{g} \mathrm{L}^{-1}$, and all the optimization experiments were conducted three times, with extraction recovery as the indicator of MSPD performance.

\subsubsection{Effects of washing solvent and eluent solvent}

Generally, in most real sample analysis, the concentrations of target analytes are at trace/ultra-trace level while matrix interferences commonly have the concentrations more than a thousand fold compared with analytes. This situation is very detrimental to analytical determination based on three reasons: (1) the peak intensity of the matrix is much more than the analyte peak and can interfere and/or overlap the analyte peak, (2) the sensitivity of the method can be greatly decreased, and (3) the chromatography column is easily polluted and thereby causing shorter service life. Therefore, matrix interferences should be effectively reduced and eliminated; in this study, they can be removed by a washing solvent. Washing solvents should rinse the interference compounds which are adsorbed on the sorbent, and meantime leave the analytes. In order to attain the optimal washing/extraction recovery, several solvents with varying polarities were tested. As shown in Fig. $\mathrm{S} 3$, the extraction recoveries of $\mathrm{RhB}$ using n-hexane, ethyl acetate, acetone, deionized water and ethanol were $92 \%, 83 \%, 53 \%, 46 \%$, and $40 \%$, respectively. Because $\mathrm{n}$-hexane has high strength to dissolve nonpolar interferents, it can easily remove hydrophobic matrix-interferences. However, RhB is hydrophilic and insoluble in nonpolar solvents (e.g., n-hexane), so that it was retained on the MINs sorbent. Also, it can be deduced that most of the matrix impurities were hydrophobic compounds and can easily dissolve in n-hexane. Thus, n-hexane was used for further experiments to eliminate adverse matrix effects.

Desorption of the analyte is as important as adsorbing it. Remaining a few analyte molecules into the MINs after eluting would lead to negative error in the accuracy. The qualified eluent solvent should have high rinsing strength, polarity like RhB, being compatible with HPLC, and lower toxicity for the environment. By considering the mentioned traits, different solvents and their mixture were examined, namely acetone, methanol, ethanol, methanol/ethanol (50:50, v/v), methanol/ acetic acid (90:10, v/v), and ethanol/acetic acid (90:10, v/v). Among them, methanol/acetic acid (90:10, v/v) and ethanol/acetic acid (90:10, v/v) had the higher extraction efficiency. The addition of acetic acid to polar solvents like methanol and ethanol can hamper the formation of hydrogen bonding among RhB and APTMS and detach RhB from MINs easily. Because ethanol is classified as a green solvent, ethanol/acetic acid (90:10, v/v) was used for the experiments.

\subsubsection{Variable effects evaluation via central composite design (CCD) and response surface}

Optimization is a critical step in an analytical process since the accuracy and precision of analytical results extremely depend on the levels of the variables selected during experiments. In the traditional optimization approach (one-factor-at-a-time), the influence of the variables on the response is evaluated individually. Although this method is facile and vastly applied for both simple and complex systems, it is very difficult, tedious, and even incapable to find a true optimum level. In other words, in the analytical process several local optimum levels instead of the global optimum levels are possibly wrongfully selected as the final optimum levels. Also, the contribution of each variable to the response cannot be studied by the one-factor-ata-time method. The mentioned defects of traditional optimization can be impressively overcome by merging analytical and statistics science namely "multivariate optimization" [30].

Indeed, multivariate optimization based on central composite design (CCD) offers exclusive privileges such as fewer experiments and less time, cost, labor effort consumption as well as lower amounts of chemical waste production, comparing with other optimization methods [31]. Thus, experimental design methodology as interdisciplinary science can serve scientists to expand the first and sixth clause principles of green chemistry (Fig. S4) [32]. The function of this method is that several experiments are smartly predesigned and afterward the statistically significant relevance is found between the experimental results. Hereupon, the correlation between statistical and empirical data is a criterion for the meaningfulness of the model. In the current work, according to the background knowledge and preliminary tests, three main variables, including sample-to-dispersant ratio (A), washing solvent volume (B), and eluent solvent volume (C) were considered to design five levels $(-\alpha,-1,0,+1$ and $+\alpha)$ rotatable model consisting of 15 experimental runs (Table S3). Corresponding discussion, equations (Eqs. S1-7) and data (Fig. S5-8 and Table S4) were given in the Supporting Information. The following experimental conditions were found to give the best results: (A) sample-to-dispersant ratio $=1 / 2$, (B) washing solvent volume $=3 \mathrm{~mL}$, and (C) eluent solvent volume $=3 \mathrm{~mL}$. 


\subsection{Method validation of the MINs-MSPD-HPLC for RhB}

According to the Beer-Lambert law for spectroscopy detection of the organic compounds, UV-Vis detector should be set at a maximum wavelength because the detector has a linear response between concentration versus absorbance in this condition and also highest sensitivity is achieved. Besides, in HPLC-multiple wavelength detector (MWD) analysis, the peak characteristic (shape, area, and width) would be entirely changed by the negligible discrepancy of detection wavelength from an optimal value to the extent of a few nanometers and eventually affect the accuracy of analytical results. Since, MWD can only detect five wavelengths per each HPLC injection, in order to fully scan both UV and Vis wavelengths, and avoid several HPLC injections, the standard solution of RhB was prepared in HPLC mobile phase and analyzed by UV-Vis spectrophotometer. As seen in Fig. S9, the maximum wavelength for RhB was observed at $546 \mathrm{~nm}$. As seen, the absorbance spectra of solvents and matrix interferences at Vis range were obviously lower than that of UV range, because most of the interference species haven't chromophore. Therefore, by setting wavelength at $546 \mathrm{~nm}$, the maximum peak area of RhB with no distraction peaks in the chromatogram was achieved.

Under the above-optimized MSPD extraction conditions and HPLC analysis conditions, linearity range, limit of detection (LOD), limit of quantification (LOQ), precision and accuracy were assessed to validate the MINs-MSPD-HPLC/MWD method for the determination of RhB from foodstuffs. A calibration curve of RhB was constructed by plotting peak area versus RhB concentration in the range of $0.5-10,000 \mu \mathrm{g} \mathrm{kg}^{-1}$ with correlation coefficients of $R^{2}>0.99$. LOD and LOQ were achieved of 0.14 and $0.48 \mu \mathrm{g} \mathrm{kg}^{-1}$, respectively, using $3 S D / m$ and $10 S D / m$, in which $S D$ is the standard deviation of the peak area of blank sample ( $n=11$ ), and $m$ is the slope of the calibration curve based on the IUPAC recommendations [33]. The reproducibility and repeatability (intraand inter-day precisions), of the developed method were estimated by spiking of blank samples at concentrations of 10,100 and $1000 \mu \mathrm{gg}^{-1}$ with a standard solution of RhB, and the results were listed in Table S5. As seen, all the relative standard deviations (RSDs) values for intra- and inter-day were lower than $3.6 \%$ with satisfactory recoveries over the range of $89.2-95.8 \%$, proving excellent reproducibility.

\subsection{Application of the MINs-MSPD-HPLC method to real samples}

Three representative kinds of food samples having intense red color including chili powder, tomato ketchup and jelly powder were selected to investigate the practical application of the developed method. First, all the three selected samples were spiked with RhB solution at $10 \mu \mathrm{g} \mathrm{kg}^{-1}$, and solvent extraction was performed as follows: $0.1 \mathrm{~g}$ of each sample was dispersed into the $3 \mathrm{~mL}$ methanol: deionized water $(50: 50, \mathrm{v} / \mathrm{v})$ and dispersed by ultrasonic waves for $15 \mathrm{~min}$ and then centrifuged for $5 \mathrm{~min}(10,000 \mathrm{rpm})$. The supernatant was filtered and injected to the HPLC to see whether an interference-free RhB peak can be attained or not, and the related chromatograms were represented in Fig. 4a-c. As seen, the RhB's peak did not emerge in any of the samples due to the low concentration of analyte and the large inverted solvent peak. Accordingly, direct analysis of RhB in foodstuff is impossible and needs influential sample treatment. Afterward, the MINs-MSPD method was utilized for the analysis of all blank samples. As observed, the impurity peaks were reduced significantly, which supported the supreme anti-interference ability of the method (Fig. 4d-f). Furthermore, for the spiked samples, the interference-free RhB peak appeared explicitly at $5 \mathrm{~min}$ (Fig. 4g-i), suggesting the high applicability of the MINs-MSPD for selective enrichment and neat extraction of RhB from complicated foodstuffs. Subsequently, a spiked recovery test at three concentration levels (low, mid, and high levels of linear range) was performed by the standard addition method and considered as a criterion of accuracy. The corresponding analytical results were represented in Table 1 . As seen, tomato ketchup and jelly powder samples contained a high amount of RhB while chili powder was free of RhB. The satisfactory recoveries in the range of 83.6-96.9\% with RSDs lower than $4.6 \%$ confirmed the quantitative analysis of $\mathrm{RhB}$ could be readily accomplished with excellent reliability and accuracy.

\subsection{Method performance comparison}

The developed MINs-MSPD based method was compared with that reported MIPs based methods for the determination of RhB in terms of synthesis conditions and analytical performance, as listed in Table S6 [34-38]. As seen, the present method attains the lowest LOD in comparison with that reported, wide linear range and high recoveries. Higher IF is offered by MIPs-SPE-HPLC [34] (7.07) than ours (4.40), and others offer lower IFs [35-38]; all the reported researches require consuming a high volume of hazardous precursors and/or organic solvents [34-38]. Moreover, temperature control and/or the need for inert gas protection in preparing MIPs make the methods sophisticated [34-38]. Although the synthesis conditions of chitosan based bulk imprinting MIPs were greener/milder [38] than others [34-37], its IF (2.98) is lower than that of our surface imprinting MINs. And the bulk MIPs require to be grounded and sieved for use, and have a higher risk of incomplete template removal. On the other hand, none of the reported methods can directly analyze solid and semi-solid samples. Solvent extraction is inevitable prior to pipette-tip SPE (PT-SPE) [38] and SPE [34] for the sample of chili powder, which spends more solvents, time and effort, resulting in higher analysis costs. In contrast, our MINs synthesis procedure is very green, and clean materials are obtained without hazardous waste. In addition, MSPD aims at solid and semi-solid samples and dispense with pre-treatment (e.g., solvent extraction) of real samples. Moreover, in the MSPD, not only the extraction and clean-up steps are simultaneously performed, but also the interactions between the analytes and sorbents are greatly enhanced. So, the MINs-MSPD has the remarkable advantages of rapidity, simplicity, few organic solvents, and cost-saving. Besides, the worthy figure of merits for several real samples certifies good practicality. Therefore, the developed MINs-MSPD-HPLC method with facility, cheapness, validity, and environmental-friendliness proves to be an ideal candidate for $\mathrm{RhB}$ determination.

\section{Conclusions}

In summary, a simple, sensitive and robust MINs based MSPD method was successfully developed for the determination of RhB in food samples coupled with HPLC. Low-cost hydrophilic MINs were rationally fabricated based on surface imprinting via the synergy of CS and sol-gel precursors in aqueous media. The MINs were designed in such a way that the synthesis condition was entirely consistent with the principles of green chemistry and the resulting materials were environmentally friendly. Hence, the straightforward, cost-effectiveness and non-toxic preparation approach permit large-scale production of MINs and great potential for commercialization. The resulting MINs were successfully utilized at the service of MSPD approach for direct selective extraction of RhB from solid and semi-solid food samples. And the MINs-MSPD possessed the efficacious merit of hazardous waste elimination. Besides, experimental design relying on statistical analysis certified and simplified multivariate optimization. Consequently, the present work suggested that by merging green and sustainable chemistry perspective in high operational MINs-MSPD method, target analytes can swiftly and accurately be analyzed on eco-friendly platforms.

\section{CRediT authorship contribution statement}

Maryam Arabi: Methodology, Data curation, Formal analysis, Writing - original draft, Writing - review \& editing, Funding acquisition. Abbas Ostovan: Supervision, Conceptualization, Methodology, Funding acquisition. Ahmad Reza Bagheri: Methodology, Data 

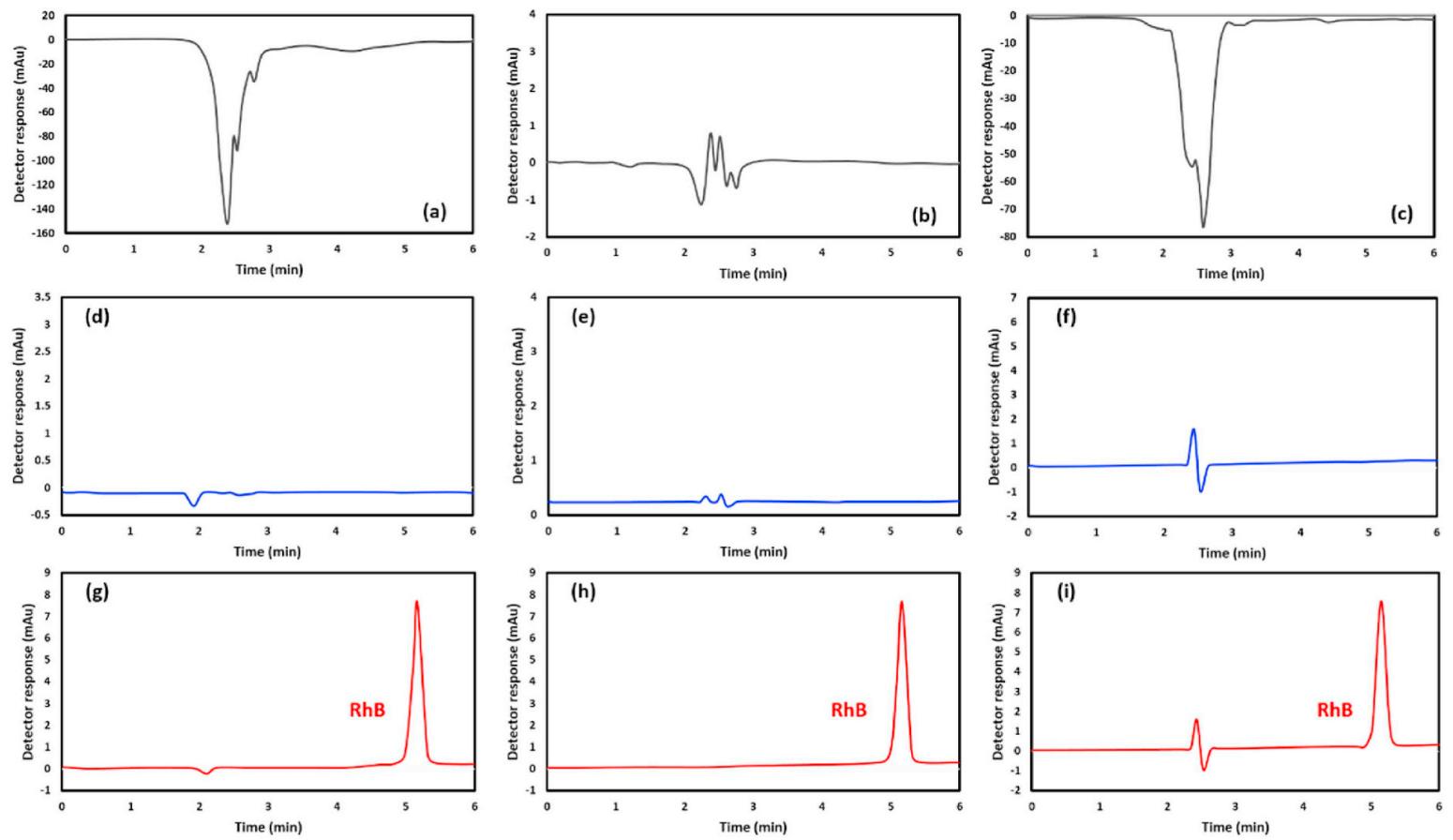

Fig. 4. Typical chromatograms of direct injection of spiked crude (a) chili powder, (b) jelly powder and (c) tomato ketchup; chromatograms of (d) chili powder, (e) jelly powder and (f) tomato ketchup after MINs-MSPD procedure without spiking; chromatograms of spiked (g) chili powder, (h) jelly powder and (i) tomato ketchup after MINS-MSPD procedure. Spiked concentration: $10 \mu \mathrm{kg}^{-1}$. HPLC conditions: deionized water-methanol (80:20, $\left.\mathrm{v} / \mathrm{v}\right)$ as mobile phase; flow rate $1.0 \mathrm{~mL}$ min ${ }^{-1}$; detection wavelength at $546 \mathrm{~nm}$; ambient temperature; Agilent C18 column (4.6 mm i.d. $250 \mathrm{~mm}, 5 \mu \mathrm{m})$.

\section{Table 1}

Endogenous contents, found values (average \pm standard deviation), relative standard deviation (RSD) and recovery of the MINs-MSPD-HPLC method for the determination of RhB in three food samples $(n=6)$.

\begin{tabular}{lllll}
\hline Food sample & $\begin{array}{l}\text { Added }(\mu \mathrm{g} \\
\left.\mathrm{kg}^{-1}\right)\end{array}$ & Found $\left(\mu \mathrm{kg}^{-1}\right)$ & $\mathrm{RSD}(\%)$ & Recovery (\%) \\
\hline Chili powder & 0 & $\mathrm{ND}^{\mathrm{a}}$ & $\mathrm{NA}^{\mathrm{b}}$ & $\mathrm{NA}$ \\
& 10 & $8.4 \pm 0.38$ & 4.6 & 83.6 \\
& 500 & $476.4 \pm 14.98$ & 3.1 & 95.3 \\
Gelly powder & 2000 & $1874.8 \pm 48.83$ & 2.6 & 93.7 \\
& 0 & $785.6 \pm 20.95$ & 2.7 & $\mathrm{NA}$ \\
& 10 & $795.2 \pm 24.60$ & 3.1 & 96.0 \\
Tomato ketchup & 0 & $1246.4 \pm 34.13$ & 2.7 & 92.2 \\
& 10 & $2723.2 \pm 88.90$ & 3.3 & 96.9 \\
& 5000 & $1386.0 \pm 43.36$ & 3.1 & $\mathrm{NA}$ \\
& 2000 & $1395.4 \pm 45.22$ & 3.2 & 94.0 \\
& 2000 & $1854.0 \pm 45.61$ & 2.5 & 93.6 \\
& & $3178.0 \pm 83.19$ & 2.6 & 89.6 \\
\hline
\end{tabular}

a Not detected.
b Not available.

curation, Validation. Xiaotong Guo: Formal analysis, Validation. Jinhua Li: Writing - review \& editing, Funding acquisition. Jiping Ma: Writing - review \& editing. Lingxin Chen: Conceptualization, Writing review \& editing, Funding acquisition.

\section{Declaration of competing interest}

The authors declare that they have no conflicts of interest with regard to the contents of this manuscript.

\section{Acknowledgments}

This work was funded by the Chinese Academy of Sciences President's International Fellowship Initiative (2019PC0050, 2020PC0083), the National Natural Science Foundation of China
(21876199, 21976099, 21976209), the Key Research and Development Plan of Shandong Province of China (GG201709290055), the Science and Technology Development Plan of Yantai City of China (2017ZH093), and the Taishan Scholar Project Special Funding (ts20190962).

\section{Appendix A. Supplementary data}

Supplementary data to this article can be found online at https:// doi.org/10.1016/j.talanta.2020.120933.

\section{References}

[1] S. Rasalingam, C.M. Wu, R.T. Koodali, Modulation of pore sizes of titanium dioxide photocatalysts by a facile template free hydrothermal synthesis method: implications for photocatalytic degradation of rhodamine B, ACS Appl. Mater. Interfaces 7 (2015) 4368-4380.

[2] A.L. Roberts, J.M. Fletcher, L. Moore, S. Byers, Trans-generational exposure to low levels of rhodamine B does not adversely affect litter size or liver function in murine mucopolysaccharidosis type IIIA, Mol. Genet. Metabol. 101 (2010) 208-213.

[3] A.L. Capriotti, C. Cavaliere, P. Foglia, R. Samperi, S. Stampachiacchiere, S. Ventura, A. Laganà, Recent advances and developments in matrix solid-phase dispersion, Trends Anal. Chem. 71 (2015) 186-193.

[4] H. Gholami, M. Ghaedi, M. Arabi, A. Ostovan, A.R. Bagheri, H. Mohamedian, Application of molecularly imprinted biomembrane for advancement of matrix solid-phase dispersion for clean enrichment of parabens from powder sunscreen samples: optimization of chromatographic conditions and green approach, ACS Omega 4 (2019) 3839-3849.

[5] L. Chen, X. Wang, W. Lu, X. Wu, J. Li, Molecular imprinting: perspectives and applications, Chem. Soc. Rev. 45 (2016) 2137-2211.

[6] L. Luo, J. Yang, K. Liang, C. Chen, X. Chen, C. Cai, Fast and sensitive detection of Japanese encephalitis virus based on a magnetic molecular imprinted polymer-resonance light scattering sensor, Talanta 202 (2019) 21-26.

[7] J. Guo, Y. Wang, Y. Liu, C. Zhang, Y. Zhou, Magnetic-graphene based molecularly imprinted polymer nanocomposite for the recognition of bovine hemoglobin, Talanta 144 (2015) 411-419.

[8] W.J. Zhang, D. Li, Y. Xu, Z. Jiang, Y. Chen, P. Wang, Synthesis and application of novel molecularly imprinted solid phase extraction materials based on carbon nanotubes for determination of carbofuran in human serum by high performance liquid chromatography, J. Agric. Food Chem. 67 (2019) 5105-5112.

[9] D. Kong, N. Wang, N. Qiao, Q. Wang, Z. Wang, Z. Zhou, Z. Ren, Facile preparation of ion-imprinted chitosan microspheres enwrapping $\mathrm{Fe}_{3} \mathrm{O}_{4}$ and graphene oxide by 
inverse suspension cross-linking for highly selective removal of copper (II), ACS Sustain. Chem. Eng. 5 (2017) 7401-7409.

[10] R. Yang, Y. Liu, X. Yan, S. Liu, H. Zheng, An effective method for the synthesis of yolk-shell magnetic mesoporous carbon-surface molecularly imprinted microspheres, J. Mater. Chem. 4 (2016) 9807-9815.

[11] Y. Fang, D. Gu, Y. Zou, Z. Wu, F. Li, R. Che, Y. Deng, B. Tu, D. Zhao, A low-concentration hydrothermal synthesis of biocompatible ordered mesoporous carbon nanospheres with tunable and uniform size, Angew. Chem. Int. Ed. 49 (2010) 7987-7991.

[12] W. Liu, L. Qin, W. Shi, L. Chen, Y. Yang, X. Liu, B. Xu, Molecularly imprinted polymers on the surface of porous carbon microspheres for capturing dibenzothiophene, Microchim. Acta 183 (2016) 1153-1160.

[13] W. Liu, H. Zhao, Y. Yang, X. Liu, B. Xu, Reactive carbon microspheres prepared by surface-grafting 4-(chloromethyl) phenyltrimethoxysilane for preparing molecularly imprinted polymer, Appl. Surf. Sci. 277 (2013) 146-154.

[14] L. Qin, X. Jia, Y. Yang, X. Liu, Porous carbon microspheres: an excellent support to prepare surface molecularly imprinted polymers for selective removal of dibenzothiophene in fuel oil, Ind. Eng. Chem. Res. 55 (2016) 1710-1719.

[15] M. Arabi, M. Ghaedi, A. Ostovan, Development of a lower toxic approach based on green synthesis of water-compatible molecularly imprinted nanoparticles for the extraction of hydrochlorothiazide from human urine, ACS Sustain. Chem. Eng. 5 (2017) 3775-3785.

[16] Z. Bie, Y. Chen, J. Ye, S. Wang, Z. Liu, Boronate-affinity glycan-oriented surface imprinting: a new strategy to mimic lectins for the recognition of an intact glycoprotein and its characteristic fragments, Angew. Chem. Int. Ed. 54 (2015) 10211-10215.

[17] A.R. Bagheri, M. Arabi, M. Ghaedi, A. Ostovan, X. Wang, J. Li, L. Chen, Dummy molecularly imprinted polymers based on a green synthesis strategy for magnetic solid-phase extraction of acrylamide in food samples, Talanta 195 (2019) 390-400.

[18] A. Ostovan, M. Ghaedi, M. Arabi, Q. Yang, J. Li, L. Chen, Hydrophilic multitemplate molecularly imprinted biopolymers based on a green synthesis strategy for determination of B-family vitamins, ACS Appl. Mater. Interfaces 10 (2018) $4140-4150$

[19] T. Zhou, L. Ding, G. Che, W. Jiang, L. Sang, Recent advances and trends of molecularly imprinted polymers for specific recognition in aqueous matrix: preparation and application in sample pretreatment, Trends Anal. Chem. 114 (2019) 11-28.

[20] M.E. Díaz-García, R.B. Laínño, Molecular imprinting in sol-gel materials: recent developments and applications, Microchim. Acta 149 (2005) 19-36.

[21] J. Lu, M. Liong, Z. Li, J.I. Zink, F. Tamanoi, Biocompatibility, biodistribution, and drug-delivery efficiency of mesoporous silica nanoparticles for cancer therapy in animals, Small 6 (2010) 1794-1805.

[22] T. Asefa, Z. Tao, Biocompatibility of mesoporous silica nanoparticles, Chem. Res. Toxicol. 25 (2012) 2265-2284.

[23] M. Atakay, O.M.R. Çelikbıçak, B. Salih, Amine-functionalized sol-gel-based lab-in- a-pipet-tip approach for the fast enrichment and specific purification of phosphopeptides in MALDI-MS applications, Anal. Chem. 84 (2012) 2713-2720.

[24] G. Zhu, G. Cheng, P. Wang, W. Li, Y. Wang, J. Fan, Water compatible imprinted polymer prepared in water for selective solid phase extraction and determination of ciprofloxacin in real samples, Talanta 200 (2019) 307-315.

[25] M.M. Titirici, M. Antonietti, Chemistry and materials options of sustainable carbon materials made by hydrothermal carbonization, Chem. Soc. Rev. 39 (2010) $103-116$.

[26] H. Freundlich, Über die adsorption in lösungen, Z. Phys. Chem. 57 (1907) 385-470.

[27] R.J. Umpleby, S.C. Baxter, Y. Chen, R.N. Shah, K.D. Shimizu, Characterization of molecularly imprinted polymers with the Langmuir-Freundlich isotherm, Anal. Chem. 73 (2001) 4584-4591.

[28] J. Hamacek, C. Piguet, How to adapt Scatchard plot for graphically addressing cooperativity in multicomponent self-assemblies, J. Phys. Chem. B 110 (2006) 7783-7792.

[29] Y. Huang, J. Pan, Y. Liu, M. Wang, S. Deng, Z. Xia, A Novel SPE method with two MIPs by two steps for improving selectivity of MIPs, Anal. Chem. 91 (2019) 8436-8442.

[30] S. Lin, J.K. Bediako, M.H. Song, J.A. Kim, C.W. Cho, Y. Zhao, J.W. Choi, Y.S. Yun, Effective recovery of Pt (IV) from acidic solution by a defective metal-organic frameworks using central composite design for synthesis, ACS Sustain. Chem. Eng. 7 (2019) 7510-7518.

[31] S. Wang, R. Zhang, X. Song, M. Wei, T. Xie, J. Cao, Mechanochemical-assisted extraction of active alkaloids from plant with solid acids, ACS Sustain. Chem. Eng. 7 (2018) 197-207.

[32] P.T. Anastas, J.C. Warner, Green Chemistry: Theory and Practice, Oxford University Press, 1998, p. 30.

[33] A.M. Committee, Recommendations for the definition, estimation and use of the detection limit, Analyst 112 (1987) 199-204.

[34] H. Zhai, L. Huang, Z. Chen, Z. Su, K. Yuan, G. Liang, Y. Pan, Chip-based molecularly imprinted monolithic capillary array columns coated $\mathrm{GO} / \mathrm{SiO}_{2}$ for selective extraction and sensitive determination of rhodamine $\mathrm{B}$ in chili powder, Food Chem. 214 (2017) 664-669.

[35] X. Liu, D. Yu, Y. Yu, S. Ji, Preparation of a magnetic molecularly imprinted polymer for selective recognition of rhodamine B, Appl. Surf. Sci. 320 (2014) 138-145.

[36] Z. Long, W. Xu, Y. Lu, H. Qiu, Nanosilica-based molecularly imprinted polymer nanoshell for specific recognition and determination of rhodamine B in red wine and beverages, J. Chromatogr. B 1029 (2016) 230-238.

[37] Z. Long, W. Xu, Y. Peng, Y. Lu, Q. Luo, H. Qiu, Highly selective coextraction of rhodamine B and dibenzyl phthalate based on high-density dual-template imprinted shells on silica microparticles, J. Separ. Sci. 40 (2017) 506-513.

[38] A.R. Bagheri, M. Ghaedi, Synthesis of chitosan based molecularly imprinted polymer for pipette-tip solid phase extraction of Rhodamine B from chili powder samples, Int. J. Biol. Macromol. 139 (2019) 40-48. 